\title{
Evaluación de un programa grupal de educación para progenitores: impacto en las competencias parentales
}

\author{
Silvia Vaca Gallegos \\ Universidad Técnica Particular de Loja, Ecuador \\ mail: slvacadutpl.edu.ec \\ ORCID: https://orcid.org/0000-0001-6333-0267
}

\author{
Aranzazu Cisneros Vidal \\ Universidad Técnica Particular de Loja, Ecuador \\ mail: macisnerosautpl.edu.ec \\ ORCID: https://orcid.org/0000-0002-8109-4760
}

\author{
Nairoby Jackeline Pineda Cabrera \\ Universidad Técnica Particular de Loja, Ecuador \\ mail: njpinedadutpl.edu.ec \\ ORCID: https://orcid.org/0000-0001-9512-4900
}

\section{RESUMEN}

Los programas de intervención para progenitores son cada vez más relevantes a la hora de promover, prevenir y tratar la salud mental en adolescentes, desde la responsabilidad de apoyar a sus hijos e hijas en el desarrollo de habilidades para una adultez funcional y exitosa. Por ello, el objetivo de esta investigación es aplicar y determinar la eficacia del Programa para el Desarrollo de Competencias Emocionales, Educativas y Parentales (Martínez González, 2009) en padres y madres con hijos e hijas adolescentes de la ciudad de Loja, Ecuador. La metodología utilizada fue a través de un método experimental, con enfoque cuantitativo y temporalidad retrospectiva y prospectiva; participaron 162 progenitores o representantes, se aplicó el Cuestionario de evaluación inicial de competencias parentales y se implementó el Programa-Guía para el desarrollo de competencias emocionales, educativas y parentales (Martínez et al., 2009) y, se desarrollaron análisis estadísticos de doble sentido, tanto estadísticos descriptivos como comparaciones paramétricas a través de análisis de la varianza (ANOVA), además, se analizó la consistencia interna del cuestionario de evaluación inicial de competencias parentales mediante el coeficiente de confiabilidad alfa de Cronbach.

La investigación concluye que mediante este programa se obtienen cambios significativos en cinco (necesidad de atención, respeto, afecto y reconocimiento; autoestima y asertividad; comunicación asertiva; resolución de problemas y disciplina, normas, reglas) de las seis dimensiones evaluadas e intervenidas, dentro del grupo experimental (pre-post), así como diferencias significativas entre el grupo experimental y el grupo control. En cuanto a la mejora de las dimensiones, la mayoría de los progenitores afirman haber desarrollado mejores competencias en todas las dimensiones analizadas, por ejemplo, una que obtiene mayor percepción de mejora es la de autoestima y asertividad (80,6\%), en la misma se logró afianzar la confianza en sí mismos como progenitores.

Palabras Clave: parentalidad positiva, educación parental, programas de educación parental, adolescentes, salud mental.

\section{Evaluation of a group-based parenting education program: impact on parenting competencies}

\section{ABSTRACT}

Intervention programs for parents are becoming increasingly relevant when it comes to promoting, preventing, and treating mental health in adolescents, based on their responsibility to support their children in the development of skills for a functional and successful adulthood. Therefore, the objective of this research is to apply and determine the effectiveness of the Program for the Development of Emotional, Educational and Parental Competencies (Martínez González, 2009) in fathers and mothers with adolescent sons and daughters in the city of Loja, Ecuador. The methodology used was an experimental method, with a quantitative approach and retrospective and prospective temporality. In this program, 162 parents or representatives participated, the initial evaluation questionnaire of parental competencies was applied, and the Program-Guide for the development of emotional, educational and parental competencies was implemented (Martínez et al., 2009). Two-way statistical analyses were developed-both descriptive statistics and parametric comparisons through analysis of variance (ANOVA). In addition, the internal consistency of the initial evaluation questionnaire of parental competencies was analyzed through Cronbach's alpha reliability coefficient.

ISSN: 0210-2773

DOI: https://doi.org/10.17811/rifie.50.4.2021.799-806 
The research concludes that, through this program, significant changes are obtained in five (need for attention, respect, affection, and recognition; self-esteem and assertiveness; assertive communication; problem solving and discipline, norms, rules) of the six dimensions evaluated and intervened, within the experimental group (pre-post), as well as significant differences between the experimental group and the control group. Regarding the improvement of the dimensions, most of the parents claim to have developed better competences in all the dimensions analyzed. For instance, one that obtains a higher perception of improvement is that of self-esteem and assertiveness (80.6\%), in which they were able to strengthen their self-confidence as parents.

Keywords: positive parenting, parental education, parenting education programs, adolescents, mental health.

\section{Introducción}

Los programas de intervención para padres y medres cada vez tienen más relevancia a la hora de promocionar, prevenir y tratar la salud mental en los adolescentes, desde el supuesto de la responsabilidad de apoyar a sus hijos e hijas en el desarrollo de habilidades en proyección de una adultez funcional y exitosa; así como también proporcionar un entorno seguro, amoroso y cálido, caracterizado por una supervisión eficaz que expone límites claros, permitiendo a los adolescentes crecer, aprender, fallar y triunfar (Costantini et al., 2020).

Vila (1998) manifiesta que la formación de progenitores es "un conjunto de actividades voluntarias de aprendizaje por parte de los progenitores que tiene como objetivo proveer modelos adecuados de prácticas educativas en el contexto familiar y/o modificar y mejorar prácticas existentes con el objeto de promover comportamientos en los hijos e hijas que son juzgados positivamente y erradicar los que se consideran negativos" ( $\mathrm{p}$. 502). Cuando esta formación se desarrolla en el marco escolar contribuye a fomentar relaciones de cooperación entre los dos microsistemas fundamentales para el desarrollo personal de los menores: la familia y el centro docente (Bronfenbrenner, 1979; Martínez González et al., 2000; Martínez González et al., 2004; Martínez González y Rodríguez Ruiz, 2007).

Los resultados de un metaanálisis (Yap et al., 2016), reporta que las intervenciones preventivas dirigidas a los progenitores se asociaron con mejoras sostenidas en el bienestar de los niños y que su efectividad se prolongó hasta 11 años después de la intervención. Comparando el número de intervenciones dirigidas a padres y madres de niños pequeños se resalta pocas intervenciones disponibles para los progenitores de adolescentes (Wade et al., 2019), por lo que esta investigación aporta con resultados para esta etapa evolutiva.

Los resultados de intervenciones con padres y madres de adolescentes muestran una mejora en el bienestar de los adolescentes, en la dinámica familiar y en las relaciones entre padres, madres, hijos e hijas (Kuhn y Laird, 2014; Yap et al., 2016; Chu et al., 2015; Chand et al., 2013). La paternidad eficaz es un factor protector contra algunos efectos negativos que pueden suscitarse en esta etapa, entre ellas, el absentismo escolar y las dificultades asociadas a conductas de riesgo (Wang et al., 2011), así como la experiencia sexual temprana, el consumo de alcohol y otras drogas (Kelly et al., 2011).

Otras revisiones sistemáticas sobre el desarrollo positivo de la parentalidad han identificado ciertas características psicológicas y emocionales que promueven la salud mental y el desarrollo social en los adolescentes. Las más identificadas están asociadas a la auto-regulación y autoestima, el afrontamiento y la perseverancia, la responsabilidad y la toma de decisiones, la resolución de problemas, la motivación y el logro con orientación al futuro (Arnett, 2000; Ciocanel et al., 2017; Curran y Wexler, 2017; García-Poole et al., 2019; Lerner et al. 2009; O’Connell et al., 2009; Sardiñas et al., 2017).
La adolescencia es una etapa única y formativa, pues los múltiples cambios físicos, emocionales y sociales que presentan en este periodo, incluida la exposición a la pobreza, el abuso o la violencia, pueden hacer que los adolescentes sean vulnerables a problemas de salud mental. La mitad de los trastornos mentales comienzan a los 14 años o antes, los cuales en su mayoría no se detectan ni se tratan, por lo que el suicidio se convierte en la tercera causa de muerte en los jóvenes (OMS, 2020). De acuerdo a la UNICEF (2019) más del 20\% de adolescentes en todo el mundo sufren trastornos mentales, sin embargo, estos no son diagnosticados ni tratados adecuadamente, además se estima que alrededor del 75\% de los suicidios de este grupo etario pertenecen a países de ingresos bajos y medios (Cornejo-Ortega et al., 2018).

Tedros Adhanom Ghebreyesus, director general de la OMS, manifiesta que "muy pocos niños tienen acceso a programas que les enseñen a gestionar emociones difíciles", así como también "muy pocos niños con enfermedades mentales tienen acceso a los servicios que necesitan. Esto debe cambiar" (UNICEF, 2019, párr. 6).

Es por ello que la presente investigación se focaliza en la formación de los progenitores, como actores principales del proceso de orientación requerido en la adolescencia, considerada una de las etapas más importantes del desarrollo humano, en la que se producen cambios físicos, emocionales y sociales. Esta etapa involucra además una serie de responsabilidades, pero también de inseguridades y desafíos, constituyéndose así en una etapa fundamental para el individuo y su familia.

De ahí la importancia del presente estudio, que tiene por objetivo aplicar y determinar la eficacia del Programa para el Desarrollo de Competencias Emocionales, Educativas y Parentales (Martínez González, 2009) en padres y madres con hijos e hijas adolescentes de la ciudad de Loja (Ecuador). Este programa ha sido revelado como una de las acciones formativas grupales de educación parental más evaluadas e implementadas a nivel internacional (Rubio et al., 2020).

\section{Metodología}

\section{Diseño y tipo de investigación}

La presente investigación se basa en las características de ser experimental, con un enfoque cuantitativo y temporalidad retrospectiva y prospectiva.

\section{Participantes}

En el estudio participaron 162 padres, madres o representantes, distribuidos en un grupo experimental (a quienes se aplicó el programa) y otro grupo de control, seleccionados a través de un muestreo no probabilístico por conveniencia o incidental, que permitió seleccionar una muestra de la población por el hecho de ser accesible (Blanco, 2007). 
Como criterios de inclusión constaban el tener un hijo(a) adolescente asistiendo a algún nivel de la educación general básica de un establecimiento educativo diurno de la zona urbana de la ciudad de Loja y aceptar el consentimiento informado, en el cual se incluyó una descripción del estudio con su respectiva introducción, el propósito, los procedimientos, los riesgos y beneficios y la confidencialidad de los datos; se especificaron las medidas necesarias que se aplicarían para proteger la identidad de los participantes y la custodia de sus datos, derechos y opciones, la información de contacto y finalmente el consentimiento informado para que el padre, madre o representante del menor de edad colocase su respectiva firma y fecha. Además, es importante señalar que todos los estándares éticos declarados en Helsinski (Williams, 2008) con respecto a la investigación de seres humanos se encontraron presentes durante esta investigación.
Las principales características sociodemográficas de los padres, madres o representantes del grupo experimental fueron las siguientes: el 65\% fueron mujeres, el 44,4\% tenían estudios universitarios, seguido de estudios secundarios (17,3\%), el 72,8\% tenía como estado civil el de casados y un $13,6 \%$ divorciados. En relación con la estructura familiar, 1 67,5\% eran familias de tipo nuclear, seguido de la familia monoparental (17,5\%).

\section{Instrumentos}

Se utilizó el Cuestionario de evaluación inicial de competencias parentales (Martínez, 2009), aplicado en la primera sesión del programa "Guía para el desarrollo de competencias emocionales, educativas y parentales" que consta de tres apartados que se describen en la Tabla 1.

Tabla 1

Cuestionario de evaluación inicial

\begin{tabular}{|c|c|c|}
\hline Dimensiones & $N^{\circ}$ Preguntas & Indicadores \\
\hline Información Sociodemográfica & 11 & $\begin{array}{l}\text {-Sexo } \\
\text {-Edad } \\
\text {-Estudios } \\
\text {-Nacionalidad } \\
\text {-Lugar de procedencia } \\
\text {-Estado civil } \\
\text {-Tipología familiar } \\
\text {-Situación laboral }\end{array}$ \\
\hline Competencias parentales & 16 & $\begin{array}{l}\text {-Necesidad de atención, respeto, afecto y reconocimiento. } \\
\text {-Autoestima y asertividad. } \\
\text {-Comunicación asertiva } \\
\text {-Resolución de problemas } \\
\text {-Disciplina, normas y reglas }\end{array}$ \\
\hline Relaciones entre padres e hijo(a)s & 25 & $\begin{array}{l}\text {-Características evolutivas del menor } \\
\text {-Habilidades cognitivas de relajación y autorregulación }\end{array}$ \\
\hline
\end{tabular}

Otros aspectos importantes del cuestionario tienen relación con los siguientes aspectos: el objetivo que pretende el cuestionario al ser aplicado en un primer momento, en la evaluación inicial, con la finalidad de socializar los contenidos del programa e identificar las necesidades de formación para el desarrollo adecuado del rol parental, así como también para la evaluación final.

Otro aspecto importante es el tiempo aproximado para completarlo, que es entre 25 a 35 minutos. Puede ser de aplicación individual o colectiva y en formato impreso. Las preguntas son cerradas en su mayoría (medidas en escala de tipo Likert con 3 opciones de respuesta) y algunas abiertas. En esta investigación se ha realizado un análisis solo de las primeras.

En cuanto a la forma de calificación se procede a obtener puntajes en relación a cada dimensión y un puntaje global, puntajes que se describen en la Tabla 2. Sin embargo, es menos conveniente utilizar el puntaje global para identificar el nivel de competencias parentales por el constructo que se trabaja, siendo más útil obtener los puntajes por cada una de las dimensiones, para lo cual se elabora una ficha de registro similar a un perfil de competencias parentales.

Se implementó el Programa-Guía para el desarrollo de competencias emocionales, educativas y parentales (Martínez et al., 2009), el cual es un programa de prevención primaria que intenta responder a las necesidades de asesoramiento educativo manifestadas por padres y madres.
El programa incluye las siguientes acciones: 1) mejorar el conocimiento de los padres y madres de familia sobre las características evolutivas de sus hijos e hijas, 2) desarrollar estrategias sobre estilos educativos positivos (educación familiar) y 3) facilitar y afrontar las dificultades y conflictos cotidianos que se producen en la familiar.

Este programa constituye una medida de apoyo socioeducativo dirigido a familias, con independencia de su tipología, estructura, dinámica interna o ciclo vital; no discrimina entre familias normalizadas o en situaciones especiales (desfavorecidas o en riesgo); con un enfoque fundamentalmente preventivo, comunitario y basado, entre otras, en dos estrategias fundamentales: fomentar los puntos fuertes y las potencialidades de las familias y de las personas, evitando centrarse solo en sus limitaciones y carencias (McKnight, Kretzmann, 1990).

\section{Procedimiento}

La presente experiencia partió del proyecto de investigación "Desarrollo de competencias emocionales, educativas y parentales en la ciudad de Loja" (Aprobación MSP, Oficio No. MSPVGVS-2016-0244-O, 26 de abril de 2016) aprobado por el Comité de Ética de Investigación en Seres Humanos, Universidad San Francisco de Quito, Ecuador para su ejecución. Con el fin de organizar los distintos momentos de la aplicación de este programa se exponen las fases desarrolladas. 
Figura 1.

Fases del Programa. Nota: Martínez González, 2009

Primera Fase-Contacto con centros educativos donde se realizó la intervención. En el caso de que acepten participar se solicitó una carta de compromiso de colaboración.

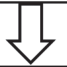

Segunda Fase-Evaluación y presentación del programa. Entrega del consentimiento informado, de aceptar la participación se aplicó el cuestionario sociodemográfico y el pre-test de competencias parentales.

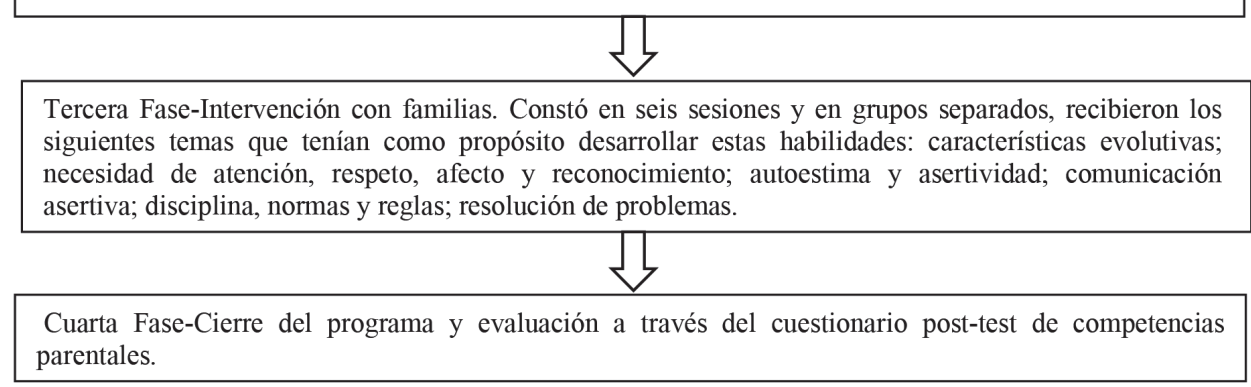

Nota: Martínez González, 2009

\section{Análisis estadísticos}

Se desarrollaron análisis estadísticos en un doble sentido, por un lado, se procedió al cálculo de los estadísticos descriptivos para conocer los resultados obtenidos del cuestionario y las variables sociodemográficas. Por otro lado, se realizaron las comparaciones paramétricas pertinentes entre el grupo control y experimental, a través de análisis de la varianza (ANOVA) que permite comparar las medias para datos independientes. También se analizó la consistencia interna del cuestionario de evaluación inicial de competencias parentales (Martínez, 2009), mediante el coeficiente de confiabilidad alfa de Cronbach.

\section{Resultados}

Luego de aplicar el Alfa de Cronbach, se comprobó que el cuestionario utilizado tiene una fiabilidad de 0,83 (43 elementos), a partir de este valor y considerando la clasificación de los niveles de fiabilidad según Avecillas y Lozano (2016), se concluyó que el valor obtenido indica que el instrumento tiene un muy buen nivel de fiabilidad.

Se evidencia que entre el grupo control y grupo experimental existen diferencias en las medias, en el cuestionario de competencias parentales del pos-test, se observa una mejora en las cinco dimensiones a excepción de las características evolutivas, que

Tabla 2

Comparación de medias entre grupo control y experimental en el post-test

\begin{tabular}{|c|c|c|c|c|c|c|c|c|c|}
\hline & & \multirow[t]{2}{*}{$\mathrm{N}$} & \multirow[t]{2}{*}{ Media } & \multirow[t]{2}{*}{$\begin{array}{l}\text { Desviación } \\
\text { estándar }\end{array}$} & \multirow[t]{2}{*}{$\begin{array}{l}\text { Error } \\
\text { estándar }\end{array}$} & \multicolumn{2}{|c|}{$\begin{array}{c}95 \% \text { del intervalo } \\
\text { de confianza } \\
\text { para la media }\end{array}$} & \multirow[t]{2}{*}{ Mínimo } & \multirow[t]{2}{*}{ Máximo } \\
\hline & & & & & & Límite inferior & Límite superior & & \\
\hline \multirow{3}{*}{$\begin{array}{l}\text { Necesidad de atención, } \\
\text { respeto, afecto y reco- } \\
\text { nocimiento PT }\end{array}$} & Experimental & 32 & 23,78 & 3,07 & ,54 & 22,67 & 24,89 & 18 & 30 \\
\hline & Control & 28 & 21,14 & 2,90 &, 54 & 20,01 & 22,26 & 18 & 28 \\
\hline & Total & 60 & 22,55 & 3,25 & ,42 & 21,70 & 23,39 & 18 & 30 \\
\hline $\begin{array}{l}\text { Autoestima y asertivi- } \\
\text { dad PT }\end{array}$ & Total & 59 & 28,57 & 4,14 &, 53 & 27,49 & 29,65 & 18,00 & 37 \\
\hline \multirow{3}{*}{$\begin{array}{l}\text { Comunicación asertiva } \\
\text { PT }\end{array}$} & Experimental & 32 & 32,50 & 3,29 &, 58 & 31,31 & 33,68 & 24,00 & 38 \\
\hline & Control & 28 & 27,71 & 4,89 & ,92 & 25,81 & 29,61 & 16 & 38 \\
\hline & Total & 60 & 30,26 & 4,74 & 61 & 29,04 & 31,49 & 16 & 38 \\
\hline $\begin{array}{l}\text { Resolución de proble- } \\
\text { mas PT }\end{array}$ & Experimental & 33 & 14,87 & 1,81 & 31 & 14,23 & 15,52 & 9 & 19 \\
\hline $\begin{array}{l}\text { Disciplina, normas, } \\
\text { reglas PT }\end{array}$ & Total & 60 & 19,58 & 2,85 & ,36 & 18,84 & 20,32 & 13 & 26 \\
\hline \multirow{3}{*}{$\begin{array}{l}\text { Características evoluti- } \\
\text { vas del menor PT }\end{array}$} & Experimental & 33 & 2,27 &, 83 & ,14 & 1,97 & 2,57 & 1,00 & 4,00 \\
\hline & Control & 28 & 2,57 & ,74 & ,14 & 2,28 & 2,85 & 1,00 & 4,00 \\
\hline & Total & 61 & 2,40 &, 80 & ,10 & 2,20 & 2,61 & 1,00 & 4,00 \\
\hline
\end{tabular}


las medias son muy parecidas. Cabe indicar que la dimensión de características evolutivas es conocimiento, y al empezar el programa la mayoría de los padres, madres o representantes indicaron que conocen muchas de las características de sus hijos e hijas.

Tabla 3

Diferencias entre grupos. (ANOVA)

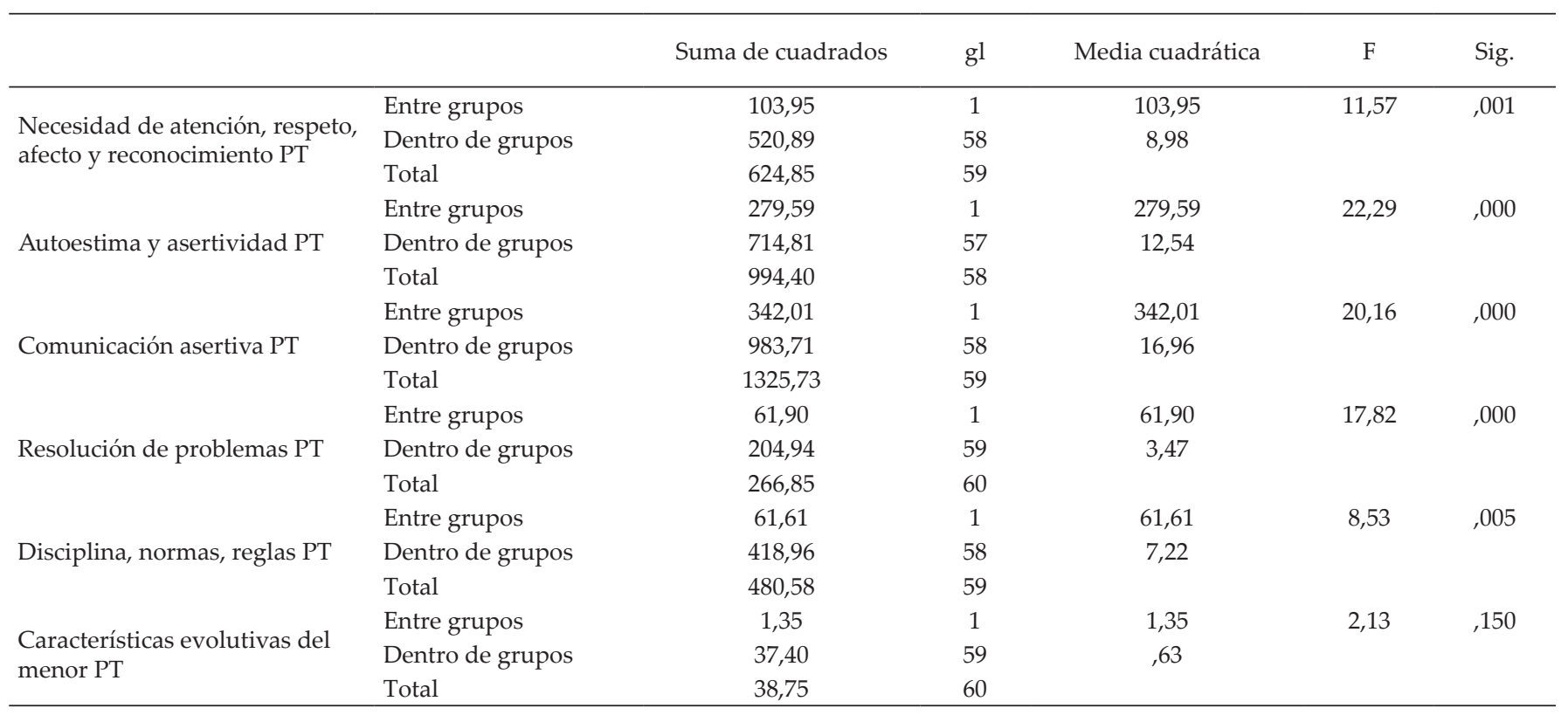

Nota: PT= Post-test del Cuestionario de competencias parentales

Tabla 4

Comparación de mejora entre el grupo experimental y grupo control

\begin{tabular}{|c|c|c|c|c|c|}
\hline \multirow{4}{*}{$\begin{array}{l}\text { Necesidad de atención, respeto, afecto } \\
\text { y reconocimiento }\end{array}$} & & \multicolumn{4}{|c|}{ Grupo } \\
\hline & & $\mathrm{N}$ & $\begin{array}{c}\text { Experimental } \\
\% \text { columna }\end{array}$ & $\mathrm{N}$ & $\begin{array}{l}\text { Control } \\
\% \text { columna }\end{array}$ \\
\hline & Mantiene & 2 & $6,3 \%$ & 6 & $21,4 \%$ \\
\hline & Mejora & 22 & $68,8 \%$ & 11 & $39,3 \%$ \\
\hline & Empeora & 8 & $25,0 \%$ & 11 & $39,3 \%$ \\
\hline & Mantiene & 1 & $3,2 \%$ & 3 & $10,7 \%$ \\
\hline \multirow[t]{3}{*}{ Autoestima y asertividad } & Mejora & 25 & $80,6 \%$ & 15 & $53,6 \%$ \\
\hline & Empeora & 5 & $16,1 \%$ & 10 & $35,7 \%$ \\
\hline & Mantiene & 1 & $3,1 \%$ & 3 & $10,7 \%$ \\
\hline \multirow[t]{3}{*}{ Comunicación asertiva } & Mejora & 22 & $68,8 \%$ & 14 & $50,0 \%$ \\
\hline & Empeora & 9 & $28,1 \%$ & 11 & $39,3 \%$ \\
\hline & Mantiene & 3 & $9,1 \%$ & 7 & $25,0 \%$ \\
\hline \multirow[t]{3}{*}{ Resolución de problemas } & Mejora & 22 & $66,7 \%$ & 9 & $32,1 \%$ \\
\hline & Empeora & 8 & $24,2 \%$ & 12 & $42,9 \%$ \\
\hline & Mantiene & 4 & $12,5 \%$ & 5 & $17,9 \%$ \\
\hline \multirow[t]{3}{*}{ Disciplina, normas, reglas } & Mejora & 21 & $65,6 \%$ & 9 & $32,1 \%$ \\
\hline & Empeora & 7 & $21,9 \%$ & 14 & $50,0 \%$ \\
\hline & Mantiene & 13 & $39,4 \%$ & 21 & $75,0 \%$ \\
\hline \multirow[t]{2}{*}{ Características evolutivas } & Mejora & 9 & $27,3 \%$ & 3 & $10,7 \%$ \\
\hline & Empeora & 11 & $33,3 \%$ & 4 & $14,3 \%$ \\
\hline
\end{tabular}

Nota: PT= Post-test del Cuestionario de competencias parentales

En cambio, en las otras dimensiones se refiere a las habilidades emocionales, educativas y parentales que se supone que los progenitores deben tener y que se puede mejorar (Ver tabla 3). 
Se observa que el grupo experimental en las cinco dimensiones relacionadas con las habilidades parentales, tienen resultados de mejora en mayor porcentaje que el grupo control, quienes en su mayoría se mantienen o empeoran. Nuevamente en la dimensión de características evolutivas del menor, no existe un porcentaje muy elevado en mejora.

\section{Discusión y conclusiones}

Al llevar a cabo el programa se obtienen cambios significativos en cinco (necesidad de atención, respeto, afecto y reconocimiento; autoestima y asertividad; comunicación asertiva; resolución de problemas y disciplina, normas, reglas) de las seis dimensiones evaluadas e intervenidas, dentro del grupo experimental (pre-post), así como también diferencias significativas entre el grupo experimental y el grupo control.

En cuanto a la mejora de las dimensiones, la mayoría de los padres afirman haber desarrollado mejores competencias en todas las dimensiones analizadas (Martínez-Gonzales et al., 2016; Vázquez et al., 2020). Por ejemplo, una de las dimensiones con las que se evidencia mayor percepción de mejora es la de la autoestima y asertividad, varios estudios señalan la importancia de la influencia que asertividad, la autoestima, el locus de control y el comportamiento de los padres tienen en la presencia de comportamientos de riesgo en adolescentes (Janković, U.,2021). De esta forma, se logró afianzar la confianza en sí mismos como padres para luego adaptarse y ganar la confianza de sus hijos (Martínez-González, 2009).

En este sentido, el desarrollo de esta competencia les permite tener una visión más positiva antes las dificultades que se puedan presentar y evitar la culpabilidad por sus acciones (Martínez-González et al., 2016). Asimismo, tomando en cuenta que cuando los padres adquieren competencias afectivas, comunicativas y normativas, contribuyen a la autonomía de sus hijos, siendo los adolescentes los mayormente favorecidos puesto que serán conscientes de hasta dónde pueden llegar con sus demandas y actuaciones (Urbano et al., 2018), no obstante, se ha evidenciado que mientras mayor edad tienen los hijos, existe menor formación o demanda de educación parental (Rubio et al., 2021).

Así también, se presenta una mejora en relación a la comunicación asertiva y la capacidad de identificar la necesidad de atención, respeto, afecto y reconocimiento por parte de los padres en sus hijos, significa que los asistentes reconocieron una mejora en las habilidades de empatía, valoración sobre sí mismos y sus hijos, la cooperación, respeto y autorregulación emocional (Martínez-González, 2009). Lo que es comparable a los resultados obtenidos en otros programas aplicados (Leijeten et al., 2012; Martínez-González et al., 2016), en los que también se identificó una mejora en todas las habilidades, así como en la capacidad de responder regulada y conscientemente ante las diversas conductas y dificultades de los hijos.

La implementación de las estrategias que los padres y madres adquieren en los programas generan espacios de acuerdo, además de la adquisición de mejores herramientas para comunicar y consensuar las consecuencias ante un mal comportamiento, situación que reportan posteriormente de haber participado en programas encaminados a mejorar las competencias parentales (Leijeten et al., 2012; Martínez-González., 2016; Bodenmann et al., 2008).

Sin embargo, al realizar los análisis de la dimensión sobre las características evolutivas de sus hijos, no se presentaron cambios significativos, es decir los participantes consideraron que estas habilidades se mantuvieron estables después del programa. Probablemente debido a que en la evaluación inicial fue la dimensión que puntuó más alto, por lo tanto, los participantes partieron de mayores conocimientos y estrategias previamente adquiridas.

Cabe mencionar que una de las limitaciones de este estudio es la falta de indicadores acerca de los adolescentes, así como la percepción de los hijos e hijas ante los cambios de los progenitores. No obstante, la evidencia de varios estudios (Gershater-Molko et al., 2002; Herbert, 2000; Martínez-González et al., 2016) confirman que los padres luego de haber participado de un programa de competencias parentales, emocionales y educativas desarrollan una parentalidad más positiva y menos negligente (Porzing-Ddrummong et al., 2014, Porzing-Ddrummong et al., 2015; Mendoza et al., 2018).

Considerando la relación entre los centros educativos, las familias y las entidades comunitarias, existen muchos estudios tanto en la literatura internacional como nacional que validan esta información (Rodríguez-Ruíz, et al., 2019), por tal razón, estas conclusiones preliminares podrían servir como base para la implementación de programas grupales de educación para progenitores de población infantil y adolescente en contextos de vulnerabilidad o con características específicas como culturales, sociales o étnicas.

\section{Referencias}

Arnett, J. J. (2000). Emerging adulthood: A theory of development from the late teens through the twenties. American Psychologist, 55(5), 469-480. https://doi.org/10.1037/0003-066X.55.5.469

Arévalo Avecillas, D. X., \& Padilla Lozano, C. P. (2016). Medición de la Confiabilidad del Aprendizaje del Programa RStudio Mediante Alfa de Cronbach. Revista Politécnica, 37(1), 68. https://revistapolitecnica.epn.edu.ec/ojs2/index.php/revista_politecnica2/article/view/469

Bodenmann, G., Cina, A., Ledermann, T., y Sanders, M. R. (2008). The efficacy of the Triple P-Positive Parenting Program in improving parenting and child behavior: a comparison with two other treatment conditions. Behaviour research and therapy, 46(4), 411-427. https://doi.org/10.1016/j.brat.2008.01.001

Bronfenbrenner, U. (1979). The ecology of human development. Cambridge, Harvard University Press.

Chand, N., Farruggia, S., Dittman, C., Sanders, M., y Ting Wai Chu, J. (2013). Promoting positive youth development: through a brief parenting intervention program. Youth Studies Australia, 32(1), 29. https://search.informit.org/doi/10.3316/informit.174146305394013

Chu, J. T. W., Bullen, P., Farruggia, S. P., Dittman, C. K., y Sanders, M. R. (2015). Parent and adolescent effects of a universal group program for the parenting of adolescents. Prevention Science, 16(4), 609-620. https://doi.org/10.1007/s11121-0140516-9

Ciocanel, O., Power, K., Eriksen, A., y Gillings, K. (2017). Effectiveness of positive youth development interventions: A metaanalysis of randomized controlled trials. Journal of Youth and Adolescence, 46(3), 483-504. https://doi.org/10.1007/s10964016-0555-6

Cornejo-Ortega, A., Rojas-Omaña, A, Bonilla-Cruz, N. y Rivera-Porras, D. (2018). Estrategias de afrontamiento como elemento terapéutico en la orientación suicida de adolescentes de un colegio de la comuna 7en Cúcuta. Archivos Venezolanos de Farmacología Terapéutica, 37(5), 528-533.

Curran, T., y Wexler, L. (2017). School-based positive youth development: A systematic review of the literature. Journal of School Health, 87(1), 71-80. https://doi.org/10.1111/josh.12467

García-Poole, C., Byrne, S., y Rodrigo, M. J. (2019). How do communities intervene with adolescents at psychosocial risk? A 
systematic review of positive development programs. Children and Youth Services Review, 99, 194-209. https://doi.org/10.1016/j.childyouth.2019.01.038

Gershater-Molko, R. M., Lutzker, J. R., y Sherman, J. A. (2002). Intervention in child neglect: An applied behavioral perspective. Aggression and Violent Behavior, 7(2), 103-124. https://doi. org/10.1016/S1359-1789(00)00035-5

Herbert, M. (2000). Parenting skills interventions. In P. Reder, M. McClure, y A. Jolley (Eds.), Family matters: Interfaces between child and adult mental health (pp.237-256). Routledge

Janković, U. (2021). Asertividad, autoestima e influencia de los padres (padres) como factores en las conductas de alto riesgo de los estudiantes de secundaria: el caso de Montenegro. Sociologija , 63 (1), 50-71. https://doi.org/10.2298/SOC2101050J

Kelly, C.M., Mithen, J.M., Fischer, J.A. et al. Youth mental health first aid: a description of the program and an initial evaluation. Int J Ment Health Syst 5, 4 (2011). https://doi. org/10.1186/1752-4458-5-4

Kuhn, E. S., Phan, J. M., y Laird, R. D. (2014). Compliance with parents' rules: between-person and within-person predictions. Journal of Youth and Adolescence, 43(2), 245-256. https:// doi.org/10.1007/s10964-013-9965-x

Leijten, P., Overbeek, G., y Janssens, J. M. (2012). Effectiveness of a parent training program in (pre)adolescence: evidence from a randomized controlled trial. Journal of adolescence, 35(4), 833-842. https://doi.org/10.1016/j.adolescence.2011.11.009

Lerner, J. V., Phelps, E., Forman, Y., y Bowers, E. P. (2009). Positive youth development. In R. M. Lerner y L. Steinberg (Eds.). Handbook of adolescent psychology: Individual bases of adolescent development (pp. 524-558). John Wiley y Sons Inc. https://doi. org/10.1002/9780470479193.adlpsy001016

Martínez González, R. A., Pereira González, M., Rodríguez Díez, B., Peña Del Agua, A., Martínez Álvarez, R., García González, M. P., Donaire Rubio, B., Álvarez, A. I. y Casielles Muñoz, V. (2000). Dinamización de las relaciones familia-centro escolar a través de la formación del profesorado en este campo de actuación. Revista Española de Orientación y Psicopedagogía, 11(19), 107-120 https://doi.org/10.5944/reop.vol.11. num.19.2000.11326

Martínez González, R., Pérez Herrero, H., Peña del Agua, A., García González, M., y Martínez Álvarez, R., (2004). Fomento de las relaciones de colaboración entre las familias y el profesorado a través de un programa de desarrollo de habilidades para el uso del lenguaje en niños de edad infantil. Infancia y Aprendizaje. Journal for the Study of Education and Development, 27(4), 425-435. https://doi.org/10.1174/0210370042396977

Martínez González, R., y Rodríguez Ruiz, B. (2007). Assessing parents' satisfaction with their parental role for a more effective partnership between families and schools. En H. Phtiaka y S. Symeonidou (Ed.) (2007). Schools and families in partnership: Looking into the future. Nicosia (pp. 33-43). University of Cyprus.

Martínez-González, R. A. (2009). Programa-Guía para el desarrollo de competencias emocionales, educativas y parentales. Secretaría General Técnica, Ministerio de Sanidad y Política Social. https://bit.ly/3iAV8Ra

Martínez-González, R., Álvarez, L., Henar, M. (2010). Programa-Guía para el desarrollo de competencias emocionales, educativas y parentales. Papeles salmantinos de educación, 14. 63-88. http://hdl.handle.net/10651/19047

Martínez-González, R. A., Rodríguez-Ruiz, B., Álvarez-Blanco, L., y Becedóniz-Vázquez, C. (2016). Evidencias del fomento de la parentalidad positiva mediante el Programa-Guía para el Desarrollo de Competencias Emocionales, Educativas y
Parentales. Psychosocial Intervention, 25(2), 111-117. https:// doi.org/10.1016/j.psi.2016.04.001

McKnight, J., and Kretzmann, J. P. (1990). Mapping community capacity. Center for Urban Affairs and Policy Research, Northwestern University.

Mendoza González, B., Pedroza Cabrera, F., y Martínez Martínez, K. (2018). Prácticas de Crianza Positiva: Entrenamiento a padres para reducir Bullying. Acta De Investigación Psicológica, 4(3), 1793-1808. https://doi.org/10.1016/S20074719(14)70980-9

O'Connell, M. E., Boat, T. F. y Warner, K. E. (Eds.). (2009). Preventing mental, emotional, and behavioral disorders among young people: Progress and possibilities. The National Academies Press. https://doi.org/10.17226/12480

Organización Mundial de la Salud. (28 de septiembre de 2020). Salud mental adolescente. https://bit.ly/3a8683B

Porzig-Drummon, R., Stevenson, R. J., y Stevenson, C. (2014). The 1-2-3 Magic parenting program and its effect on child problem behaviors and dysfunctional parenting: A randomized controlled trial. Behaviour Research and Therapy, 58, 5264. https://doi.org/10.1016/j.brat.2014.05.004

Porzig-Drummond, R., Stevenson, R. J., y Stevenson, C. (2015). Prelimi- nary evaluation of a self-directed video-based 1-2-3 Magic parenting pro- gram: A randomized controlled trial. Behaviour Research and Therapy, 66, 32-42. https://doi.org/10.1016/j.brat.2015.01.003

Rodríguez-Ruiz, B., Álvarez-Blanco, L., Martínez-González, R.A., \& Epstein, J. L. (2019). Presentación del número Relación entre Centros Educativos, Familias y Entidades Comunitarias. Aula Abierta, 48(1), 7-10. https://reunido.uniovi.es/index. php/AA/article/view/13290

Rubio, F. J., Trillo, M. P. y Jiménez, C. (2020). Programas grupales de parentalidad positiva: una revisión sistemática de la producción científica. Revista de Educación, 389(3), 267-295. https://doi.org/10.4438/1988-592X-RE-2020-389-462

Rubio, F. J., Trillo, M. P. y Jiménez, C. (2020). Características de los estudios de evaluación de programas grupales de educación parental. Una revisión de alcance. Revista estudios sobre educación, 41(2), 71-105. https://doi.org/10.15581/004.41.005

Sardiñas, L. M., Padilla, V., Aponte, M., Boscio, A. M., Pedrogo, C. P., Santiago, B., Morales, Á. P., Dávila, P. T., y Cesáreo, M. S. (2017). Identification of positive youth development interventions. Revista Puertorriquena de psicologia, 28(1), 14-32. https://europepmc.org/article/med/28919943

UNICEF. (05 de noviembre de 2019). Más del 20\% de los adolescentes de todo el mundo sufren trastornos mentales. https://uni. $\mathrm{cf} / 3 \mathrm{DfKgQx}$

Urbano Contreras, A., Álvarez Blanco L., \& Iglesias García M. T. (2018). Adaptabilidad y cohesión familiar del alumnado de Educación Secundaria Obligatoria. Aula Abierta, 47(2), 237244. https://doi.org/10.17811/rifie.47.2.2018.237-244

Vázquez, N., Ramos, P., Molina, M. C., \& Artazcoz, L. (2020). Social Factors Associated with the Effectiveness of a Spanish Parent Training Program - An Opportunity to Reduce Health Inequality Gap in Families. International Journal of Environmental Research and Public Health, 17(7), 2412; https://doi. org/10.3390/ijerph17072412

Vila, I. (1998). Intervención psicopedagógica en el contexto familiar. En Rodrigo, M.J. y Palacios, J. (Comp), Familia y desarrollo humano (pp.501-519). Alianza

Wade, C., Cann, W., y Matthews, J. (2019). Introduction to special issue: parenting interventions and the mental health of children and parents. Advances in Mental Health, 17(1), 1-5. https://doi.org/10.1080/18387357.2019.1585647 
Wang H, Zhou X, Lu C, Wu J, Deng X, Hong L (2011) Problematic Internet Use in High School Students in Guangdong Province, China. PLoS ONE 6(5): e19660. https://doi.org/10.1371/ journal.pone.0019660

Williams J. R. (2008). The Declaration of Helsinki and public health. Bulletin of the World Health Organization, 86(8), 650652. https://doi.org/10.2471/blt.08.050955
Yap, M. B., Morgan, A. J., Cairns, K., Jorm, A. F., Hetrick, S. E., y Merry, S. (2016). Parents in prevention: a meta-analysis of randomized controlled trials of parenting interventions to prevent internalizing problems in children from birth to age 18. Clinical Psychology Review, 50, 138-158. https://doi.org/10.1016/j.cpr.2016.10.003 